\title{
IS IT WORTH EXAMINING EEG FUNCTION IN PEOPLE UNDERGOING ORTHOPAEDIC SURGERY?
}

DOI: 10.36740/WLek202009238

\author{
Paweł Piotr Dobrakowski ${ }^{1}$, Jarosław Szyszka \\ 'PSYCHOLOGY INSTITUTE, HUMANITAS UNIVERSITY IN SOSNOWIEC, SOSNOWIEC, POLAND \\ ${ }^{2}$ ORTHOPEDIC SURGERY DEPARTMENT, OPOLE REHABILITATION CENTER, KORFANTÓW, POLAND
}

\begin{abstract}
The vast majority of hip or knee arthroplasty finishes with improved limb functional status. Despite this, some patients do not recover; they "save" the operated limb; they cannot trust it. This also happens in patients whose range of motion and muscle strength are rated as very good. It is possible, therefore, that the causes must be located in the movement initiating centre, in the motor cortex. We can link the activation and changes in the metabolism of the cerebral cortex, e.g. during limb movement, with the changes in bioelectrical activity visible in electroencephalography (EEG). Conclusions: Our literature analysis shows that so far, no study has been conducted to check whether EEG activity changes over the sensory-motor region after lower limb arthroplasty. We propose the design of such a study with an analysis of potential difficulties that would have to be overcome by the team undertaking the challenge. The obtained data could be the basis for neurorehabilitation using the EEG biofeedback method. Effective interventions would further improve the quality of life.
\end{abstract}

KEY WORDS: EEG, lower limb, hip joint, knee joint, arthroplasty

Wiad Lek. 2020;73(9 p. II):2095-2097

More and more hip and knee arthroplasty procedures are probably associated with the ageing of the population. In 2005-2017, according to the data of the National Health Fund, 506,911 hip arthroplasty and 173,015 knee arthroplasty were performed. There is a clear tendency to increase the number of performed procedures every year [1].

Osteoarthritis, especially the hip and knee joints, is one of the leading causes of pain and reduced mobility after 45 years of age. It causes disability and reduces the quality of life [2]. There are many discrepancies between objective and subjective assessments of osteoarthritis. Therefore the frequency of occurrence in European countries, varies depending on the methodology used. It ranges from 4 to $27 \%$; the relationship with age is visible [3].

Patients undergo arthroplasty treatment and benefit from rehabilitation. The vast majority of procedures end with the improvement of the functional state of the limb [4]. Despite this, some patients do not recover completely; they "save" the operated limb; they cannot trust it. This also happens in patients whose range of motion and muscle strength are rated as very good. It is possible, that the cause is located in the movement initiating centre, in the brain motor cortex. We can link the activation and changes in the metabolism of the cerebral cortex, e.g. during limb movement, with changes in bioelectrical activity visible in electroencephalography (EEG) [5].

The first results describing the changes in the EEG signal bandwidth (decrease in alpha rhythm power) associated with the stimulus-response were described in Adolf Beck's dissertation entitled "Determination of localization in the brain and spinal cord by electrical phenomena" [6]. Quantitative studies of EEG signal modulation phenomena in response to stimuli were undertaken nearly a hundred years later. Beginning with Pfurtscheller's work, these phenomena began to be called Event-Related Desynchronization (ERD) - i.e. event-related desynchronization [7]. The term ERD refers to a decrease in the power of the bioelectric spectrum in a given EEG band.

The motor cortex of the brain is spatially organized, which means that impulses to move the limb should always come from the same areas. Cortical location of ERD patterns is the result of the somatotopic organization of the sensory and motor cortex. In this arrangement, the representation of the limb is in the primary motor cortex and is generally crystallized.

Over the sensorimotor cortex, we observe the SMR rhythms (Sensorimotor Rhythms) in the EEG recording, for example, oscillations of $8-11 \mathrm{~Hz}(\mathrm{mu})$ and $12-30 \mathrm{~Hz}$ (beta) [8].

In SMR range it is possible to register ERD or Event-Related Synchronization (ERS), which are directly related to the proportional power loss in motor execution (ME) / motor imagery (MI) of the limb or the increase in signal strength at rest. The ME task is based on the physical movement of the limbs that activate the motor cortex. It includes a change in muscle tone, spasm or flexion [9]. In turn, MI is a cognitive process based on kinesthetic imagination without muscle activity, also called "Kinesthetic Motor Imagery (KMI) [10]. The advantage of MI signals is that they are free of proprioceptive feedback, as opposed to ME tasks. 
Assessing bioelectrical activity above the sensory-motor cortex during movement (or its image) of the upper limb has been popular in recent years [11-13]. The literature on similar issues for the lower limb is sparse. This is probably because the representation area of the lower limb is deep in the longitudinal cleft within the sensory-motor cortex (Penfield and Boldrey, 1937). However, research in this direction is ongoing. It has been shown so far that the analysis of beta induction ERS or mu-beta ERD has improved the distinction between left and right foot imaging as accurately as for upper limbs [14]. To our knowledge, no literature is available on the unequivocal application of KMI tasks for the knee or hip joint except $[9,15]$. The second reason for the disproportion may be an easier limitation of motor artefacts when examining the upper limb.

Our literature analysis shows that so far, no study has been conducted to check whether EEG activity changes over the sensory-motor region after lower limb arthroplasty. We searched Pubmed, IEEE Explore and Science Direct databases for a combination of passwords: EEG, SMR, ERD, lower limb, knee joint, hip joint, and arthroplasty.

Designing such research meets many potential difficulties that would have to be overcome by the team taking on the challenge.

Interdisciplinary team. Necessarily an orthopaedic specialist who can assess the efficiency of the operated joint before surgery. A specialist in electroencephalography, an expert in quantitative and qualitative EEG analysis. Good statistical background.

Scheduling trials during which the test will be recorded. Effective arthroplasty and proper rehabilitation should result in functional improvement. It would be best to evaluate the EEG changes in dynamic tests, such as walking on the treadmill, climbing stairs, getting up from a chair. Here, however, EEG artefacts from traffic may be in the way. This is one of the most severe restrictions, blocking even EEG registrations in athletes. Numerous attempts are being made to remove motion artefacts from the raw EEG signal. One of them is the method that can be used during rhythmic gait [16]. However, we are afraid that gait in people requiring endoprosthesis surgery will not be synchronous. Maybe cutting out artefacts from gait would be possible thanks to video EEG synchronization.

Another essential choice will be the electrode system for signal registration. There are dry and wet electrodes. Dry electrodes seem very tempting for the above application. They do not require the use of special conductive gels. Thanks to this, preparation for the examination is faster and more comfortable. The gel may also flow out while the object is moving and lose its properties over time [17]. If wet electrodes were chosen, it is worth choosing a thicker gel than the standard one or consider adhesive and conductive paste for fewer artefacts from agitation.

If the above methods would not sufficiently limit EEG artefacts during dynamic tests, the use of imaginary tests could be an interesting solution. Due to the existence of a mirror neuron system, even when thinking about movement, activity within the somatosensory cortex should increase. Asking the patient to imagine, e.g. climbing stairs or balancing on a balance beam may be a good option. The importance of cerebral cortex activation in the image of locomotor tasks has already been described in several papers $[18,19]$

Preparing the survey for people with reduced mobility. It will be important to provide adequate space prepared for people with mobility disabilities. Standard sets for EEG recording are quite high. It may be necessary to prepare several stairs with a handrail. Equally important would be handrails in the vicinity of the patient during dynamic tests and patient belaying by the investigators.

The right number of patients. Since in the proposed study, all people would be after endoprosthesis, it is not necessary to calculate the minimum number of people in the sample. However, in order to be able to carry out reliable statistical analyses, at least 30 people after hip surgery and 30 people after knee surgery are needed.

It will also be important to determine if there will be left-footed people among the respondents. In people with right-sided lateralization, the left side of the sensorimotor cortex activates during dominant limb movement. The situation is complicated when a person is moving with the left limbs. In addition to activating the right side cortex, the left side cortex is also activated. This is because it is more fluent in a given traffic pattern and somehow "suggests" how to perform it most economically. Recent studies show that increased activity of the contralateral and ipsilateral cortex can be demonstrated regardless of the limb being moved [20].

In our opinion, it is worth undertaking the proposed analysis, despite many potential difficulties. The obtained data could be the basis for neurorehabilitation using the EEG biofeedback method. Effective interventions would further improve the quality of life.

We wonder if the results for patients after hip and knee surgery would be different. This can be expected even because of the difference in proprioception innervation in these joints $[21,22]$.

\section{REFERENCES}

1. Realizacja świadczeń endoprotezoplastyki stawowejw 2017 r. Narodowy Fundusz Zdrowia; 2018 Jun p. www.nfz.gov.pl.

2. Culliford DJ, Maskell J, Kiran A et al. The lifetime risk of total hip and knee arthroplasty: results from the UK general practice research database. Osteoarthr Cartil. 2012; 20(6):519-24.

3. Hunter DJ, Bierma-Zeinstra S. Osteoarthritis. Lancet. 2019; 393(10182):1745-59.

4. Bachmeier CJM, March LM, Cross MJ et al. A comparison of outcomes in osteoarthritis patients undergoing total hip and knee replacement surgery. Osteoarthritis and Cartilage. 2001;9(2):137-46.

5. Leuchter AF, Uijtdehaage SH, Cook IA et al. Relationship between brain electrical activity and cortical perfusion in normal subjects. Psychiatry Res. 1999; 90(2):125-40.

6. Beck A. Oznaczenie lokalizacyi w mózgu i rdzeniu za pomocą zjawisk elektrycznych. Kraków: Akademia Umiejętności; 1890.

7. PfurtschellerG, Aranibar A. Evaluation of event-related desynchronization (ERD) preceding and following voluntary self-paced movement. Electroencephalogr Clin Neurophysiol. 1979; 46(2):138-46. 
8. Pfurtscheller G, da Silva FL EEG Event-Related Desynchronization and Event-Related Synchronization. Oxford: University Press; 2005

9. Tariq M, Trivailo PM, Simic M. EEG-Based BCI Control Schemes for LowerLimb Assistive-Robots. Front Hum Neurosci. 2018;12:312.

10. Mokienko OA, Chervyakov AV, Kulikova SN et al. Increased motor cortex excitability during motor imagery in brain-computer interface trained subjects. Front Comput Neurosci. 2013;7:168.

11. Catrambone V, Greco A, Averta G et al. EEG Complexity Maps to Characterise Brain Dynamics during Upper Limb Motor Imagery. Conf Proc IEEE Eng Med Biol Soc. 2018; 2018:3060-3.

12. Suwannarat A, Pan-Ngum S, Israsena P. Comparison of EEG measurement of upper limb movement in motor imagery training system. Biomed Eng Online. 2018;17(1):103.

13. Yi W, Qiu S, Wang K et al. Evaluation of EEG oscillatory patterns and cognitive process during simple and compound limb motor imagery. PLOS ONE. 2014;9(12):e114853.

14. Liu Y-H, Lin L-F, Chou C-W et al. Analysis of Electroencephalography EventRelated Desynchronisation and Synchronisation Induced by Lower-Limb Stepping Motor Imagery. J Med Biol Eng. 2019;39(1):54-69.

15. Tariq M, Trivailo PM, Simic M. Detection of knee motor imagery by Mu ERD/ERS quantification for $\mathrm{BCl}$ based neurorehabilitation applications. In: 2017 11th Asian Control Conference (ASCC). 2017; pp. 2215-9.

16. Gwin JT, Gramann K, Makeig S et al. Removal of movement artifact from high-density EEG recorded during walking and running. J Neurophysiol. 2010;103(6):3526-34.

17. Lopez-Gordo MA, Sanchez-Morillo D, Valle FP. Dry EEG Electrodes. Sensors. 2014;14(7):12847-70.

18. Malouin F, Richards CL, Jackson PL et al. Brain activations during motor imagery of locomotor-related tasks: a PET study. Hum Brain Mapp. 2003;19(1):47-62.
19. Pfurtscheller G, Leeb R, Keinrath C et al. Walking from thought. Brain Res. 2006;1071(1):145-52.

20. Bundy DT, Leuthardt EC. The Cortical Physiology of Ipsilateral Limb Movements. Trends Neurosci. 2019;42(11):825-39.

21. Fridén T, Roberts D, Ageberg E et al. Review of knee proprioception and the relation to extremity function after an anterior cruciate ligament rupture. J Orthop Sports Phys Ther. 2001;31(10):567-76.

22. Kaya D, Yosmaoglu B, Doral MN. Proprioception in Orthopaedics, Sports Medicine and Rehabilitation. Springer; 2018, p. 191

\section{ORCID and contributionship}

Paweł Piotr Dobrakowski - 0000-0002-6436-2487 A-B,D-F

Jarosław Szyszka - 0000-0002-5293-8362 A-B,D-F

\section{Conflict of interest}

Authors declare no conflict of interest.

\section{CORRESPONDING AUTOR}

Paweł Piotr Dobrakowski

Psychology Institute, Humanitas University in Sosnowiec

ul. Kilińskiego 43, 41-200, Sosnowiec, Poland;

tel.: +48506018622

e-mail: paweldobrakowski@interia.pl

Received: 11.07 .2020

Accepted: 14.09 .2020 